\title{
ON A CERTAIN CLASS OF CARLEMAN OPERATORS
}

\author{
S. M. BAHRI
}

Abstract.

The Carleman integral operators play an important role in spectral theory of selfadjoint operators and have been the subject of several works such as G. I. Targonski ([14], [15]), V.B.Korotkov ([7] - [10]), M. Schreiber [13], Weidmann ([17] - [19]), J.J. Grobler [6], and recently Novitski ([11], [12]). Obtaining explicit spectral development in specific cases such as differential operators, integral, integral differential ... etc. is an important research area. The work presented here is devoted to the study of spectral properties of a certain class of Carleman integral operators (the second class) in the Hilbert space $L_{2}(X, \mu)$. Such operators have their applications in approximation theory of random variables.

In a first article [1], we introduced in the Hilbert space $L_{2}(X, \mu)$ an example of integral operators of Carleman second class defined by the kernel

$$
K(x, y)=\sum_{p=0}^{\infty} a_{p} \psi_{p}(x) \overline{\psi_{p}(y)},
$$

with $\left\{a_{p}\right\}_{p=0}^{\infty}$ is a sequence of real numbers and $\left\{\psi_{p}(x)\right\}_{p=0}^{\infty}$ an orthonormal sequence in $L_{2}(X, \mu)$ verifying for almost all $x \in X$

$$
\begin{gathered}
\sum_{p=0}^{\infty} a_{p}^{2}\left|\psi_{p}(x)\right|^{2}<\infty, \\
\sum_{p=0}^{\infty}\left|\psi_{p}(x)\right|^{2}<\infty .
\end{gathered}
$$

Then we showed the selfadjointness criterion. We have determined the necessary and sufficient conditions for these operators admit selfadjoint extensions (i.e., with defect indices equal and finite). We have concluded this article by showing a practical application of such operators.

In a second article [2], we used the Straus theory [16] to describe all generalized resolvents of an Carleman operator with defect indices (1.1) in terms of a certain set of admissible analytic functions $\omega(\lambda)$. Then we describe all the generalized spectral fonctions of these operators in terms of another set of eligible analytic functions $\omega(\lambda)$. In addition, orthogonal spectral functions (i.e., those of selfadjoint extensions) are described by constant functions $\omega(\lambda)=\varkappa$ such that $|\varkappa|=1$.

2000 Mathematics Subject Classification. Primary 45P05, 47B25; Secondary 58C40.

Key words and phrases. defect indices, integral operator, spectral theory. 
The third article [4], is devoted primarily to the characterization of the convex hull of orthogonal scalar spectral functions of a Carleman operator with defect indices (1.1).

This description allowed us, in a fourth article [5], to study the spectrum of quasi selfadjoint extensions of the same operator.

\section{REFERENCES}

[1] S.M. Bahri, On the extension of a certain class of Carleman operators, EMS, ZAA, Vol. 26, $N^{\circ} .1,57-64,2007$.

[2] S.M. Bahri, Spectral properties of a certain class of Carleman operators, EMS, Archivum Mathematicum (Brno) Tomus 43, $N^{\circ} 3,163-175,2007$.

[3] S.M. Bahri, Sur les Opérateurs Intégraux de Carleman de Seconde Classe, Thèse de Doctorat, Univ. Mostaganem, 2007.

[4] S.M. Bahri, On Convex Hull of Orthogonal Scalar Spectral Functions of a Carleman Operator, Bol. Soc. Paran. Mat. (3s.) Vol. $25 N^{\circ} 1,1-10,2008$.

[5] S.M. Bahri, On the spectra of quasi selfadjoint extensions of a Carleman operator ( submit for publication ).

[6] J.J. Grobler \& G. Swanepoel, Duality between Carleman and semi-Carleman operators in Riesz spaces, Quasteones Mathematicae, Vol.14, $N^{\circ} .1,1991$.

[7] V. B. Korotkov, On characteristic properties of integral operators with kernels of Carleman type, Sib. Math. J., 11, $N^{\circ} 1,103-127,1970$.

[8] V. B. Korotkov, Opérateurs intégraux avec les noyaux satisfaisants aux conditions de Carleman-Akhiezer, Sib. Math. J. Vol. 122, $N^{\circ} 5,1267-1269,1971$.

[9] V. B. Korotkov, Sur les opérateurs intégraux forts, Sib. Math. J. Vol.15, $N^{\circ} 3,529-545$, 1974.

[10] V. B. Korotkov, Integral operators with Carleman kernels (in Russian), Nauka, Novosibirsk, 1983.

[11] I. M. Novitskii, Integral representations of unbounded operators by arbitrarily smooth Carleman kernels, preprint 2002.

[12] I. M. Novitskii, Integral representations of closed operators as bi-Carleman operators with arbitrarily smooth Carleman kernels, 2004.

[13] M. Shreiber \& Gy. I. Targonski, Carleman and semi-Carleman operators, Proc. Amer. Math. Soc. $24,1970$.

[14] Gy. I. Targonski, On Carleman integral operators, Proc. Amer. Math. Soc., $18 N^{\circ} 3,1967$.

[15] Gy. I. Targonski, Convergence theorems derived from the theory of Carleman integral operators, Comp. Math. Vol.18, Fasc.1, 2, $148-154,1967$.

[16] A.V. Straus, Extensions and generalized resolvents of a non-densely defined symmetric operator, Math. Ussr Izv.4,179-208, 1970.

[17] J. Weidman, Strong Carleman Operators are of Hilbert-Shmidt Type, Bull. Amer. Math. Soc., 74, 1968.

[18] J. Weidman, Carleman Operators, Manuscripts Math., $N^{\circ} 2,1-38,1970$.

[19] J. Weidman, Linear Operators in Hilbert Spaces, Spring Verlag, New-York Heidelberg Berlin, 1980.

Mathematics Department, Mostaganem University, BP 227, Mostaganem, 27000, AlGÉRIE

E-mail address: bahrisidimohamed@univ-mosta.dz 